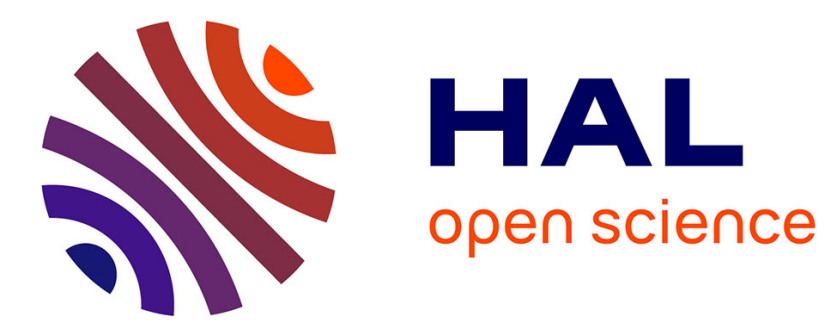

\title{
Photometric-Planner for Visual Path Following
}

Eder Rodriguez, Guillaume Caron, Claude Pegard, David Lara Alabazares

\section{To cite this version:}

Eder Rodriguez, Guillaume Caron, Claude Pegard, David Lara Alabazares. PhotometricPlanner for Visual Path Following. IEEE Sensors Journal, 2021, 21 (10), pp.11310-11317. 10.1109/JSEN.2020.3027848 . hal-02955520

\section{HAL Id: hal-02955520 \\ https://hal.science/hal-02955520}

Submitted on 2 Oct 2020

HAL is a multi-disciplinary open access archive for the deposit and dissemination of scientific research documents, whether they are published or not. The documents may come from teaching and research institutions in France or abroad, or from public or private research centers.
L'archive ouverte pluridisciplinaire HAL, est destinée au dépôt et à la diffusion de documents scientifiques de niveau recherche, publiés ou non, émanant des établissements d'enseignement et de recherche français ou étrangers, des laboratoires publics ou privés. 


\title{
Photometric-Planner for Visual Path Following
}

\author{
Eder A. Rodríguez Martínez, Guillaume Caron, Claude Pégard and David Lara-Alabazares
}

\begin{abstract}
Robotic navigation is the aspect of cognition related to robot robust mobility. It combines perception, some knowledge on the environment and a set of goal poses to reliably control the robot during a mission that involves displacement. Visionbased autonomous navigation is an instantiation of the latter discipline where visual perception is used to control the robot and to represent the environment. This paper presents a visionbased navigation system that uses its onboard camera to navigate and a visual path that represents the scene with a set of images. Being a memory-based system, the navigation is conceived as a concatenation of positioning tasks in the visual servoing scheme. The novelty on the proposed system relies on the generation of the images that compose the visual path. These images are rendered from a preobtained model of the scene. The experiments evaluate the performance of the system over three different scenes, contemplating indoor and outdoor environments, and using an Unmanned Aerial Vehicle as testbed.

Index Terms-Vision-Based Robotic Navigation, Direct Visual Servoing, Aerial Robotics.

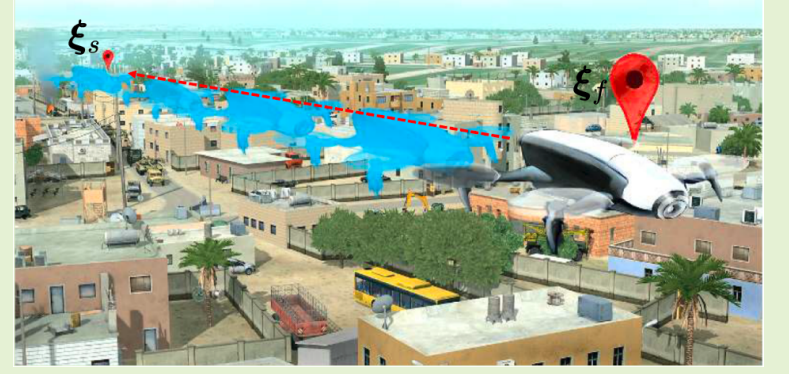

\section{INTRODUCTION}

$\mathbf{V}$ ISION-based autonomous robot navigation is the process of making the robot being able to reach a final pose, which is out of sensing range from a start pose, using vision for perception. The robot, in order to navigate from the start to the final pose, requires a path, that is usually planned offline, online or both [1]. Once the planned path is known, most of the approaches estimate the robot pose, i.e. localization, while moving, using visual odometry [2] or Simultaneous Localization And Mapping (SLAM) [3]. Then, the estimated current pose is taken as input to control the robot in order to successively follow the intermediate poses that compose the path [4]. The common scheme described above presents three distinct stages: path planning, visual perception and motion control.

The visual perception stage of the autonomous robotic navigation mostly deals with keypoints and geometric features detected and matched in images. Such geometric information can be related to the robot pose, considered in the planning as well as in the control stages. Nevertheless, robust extraction and matching of the features over a large environment is still a bottleneck for the visual navigation schemes [5]. Therefore, an alternative to the geometric information is to consider

This project was supported partially by the Mexican Council of Science and Technology, CONACYT-MEXICO.

Eder A. Rodríguez Martínez, Guillaume Caron and Claude Pégard are with the Laboratory of Modelling, Information and Systems, of the University of Picardie Jules Verne, MIS-UPJV, 33 rue Saint Leu - 80039 Amiens Cedex 1 - France (e-mail: alejandro.rodriguez@u-picardie.fr, guillaume.caron@u-picardie.fr and claude.pegard@u-picardie.fr).

David Lara-Alabazares is with the Postgraduate Department of the National Technological Institute of Mexico, Campus Misantla, TECNMITSM, Road Lomas del Cojolite Km 1.8, CO 93821 Misantla, Ver. Mexico (e-mail: dlaraa@misantla.tecnm.mx) the direct one. Indeed, a conventional camera captures light and not geometric features. However, due to the difference between the natures of photometry (visual acquisition) and geometry (3D space in which the robot moves), the direct information is less trivial to be considered for navigation. Recent progresses in direct photometric-based autonomous robot localization systems opened the way to reach similar practical performance than geometry-based approaches, and even better, since detection of geometric features and matching process are avoided [6]. Furthermore, dense methods are more precise for positioning tasks thanks to the visual feature redundancy [5]. Although implicitly, the aforementioned system directly relates two common stages of vision-based navigation: image acquisition and pose estimation (robot attitude and position). Similarly, the system proposed in [7] directly relates the stages of visual perception with motion control. This system uses Photometric Visual Servoing (PVS) [8], which considers the pixel intensities of the image as visual feature, to follow a visual path.

\section{A. Problem Statement}

On the one hand, the process of building a visual path generally starts with the acquisition of images from a first tele-operated trajectory [9]. This process requires the robot in situ and is time consuming. However, the visual path might also be planned from a preobtained dataset of images [10]. In the latter work, the visual path is represented by a weighted graph where each node is associated with an image. Then, the edges are weighted accordingly to the number of feature points matched between the images. Nevertheless, the dataset of images is required to be acquired in situ and by the same camera to ensure the point matching. 
On the other hand, the scene might be completely represented by a model, e.g. a Computer-Aided Design model [11], obtained a priori. Nevertheless, some zones may lack of texture. Moreover, localization is required when navigating.

We propose to solve these problems by generating a visual path $\mathcal{K}$ from a preobtained model of the scene. First and foremost, the model is a rectangular mesh $\mathcal{M}$, subdivided into two triangles (orange dotted lines in Fig. 11). The mesh $\mathcal{M}$ is textured by aligning its 4 vertices with those of an image of the scene, e.g. a satellite or high altitude aerial image. By using an Unmanned Aerial Vehicle (UAV) as testbed, the scene and the mesh $\mathcal{M}$ are considered flat in this paper. Secondly, the visual path $\mathcal{K}$ is composed by key images $K \in \mathcal{K}$ rendered from the mesh $\mathcal{M}$. The generation of the mesh $\mathcal{M}$ and the rendering of each key image $K$ are computed offline. Lastly, the autonomous navigation is defined as a concatenation of positioning tasks which goal is to sequentially servo each key image $K$; called visual path following.

The system proposed in this paper is presented next.

\section{B. Proposed System}

The main contribution in this paper is a vision-based navigation system composed by the Photometric-Planner and the Visual Path Follower.

The goal of the Photometric-Planner is to generate a navigable visual path $\mathcal{K}$. Generally speaking, this process begins by rendering virtual images $I_{V}$ from the mesh $\mathcal{M}$. Then a Gaussian filter is applied to these images and a Zero-mean Normalization transformation to their pixel intensities. Finally, some of these $I_{V}$ are selected; thus, they become the elements in the visual path $\mathcal{K}$.

The Visual Path Follower is in charge of carrying out the autonomous navigation. Being a memory-based system, the UAV is controlled by comparing the onboard images $I$ with those stored in the visual path $\mathcal{K}$ using a variation of PVS, called Enhanced PVS. Indeed, to servo a desired image $I^{*}$ using an UAV as testbed is a secondary contribution since this image is virtual (so, denoted as $I_{V}^{*}$ ).

The diagram in Fig. 1 illustrates the general workflow of the proposed system.

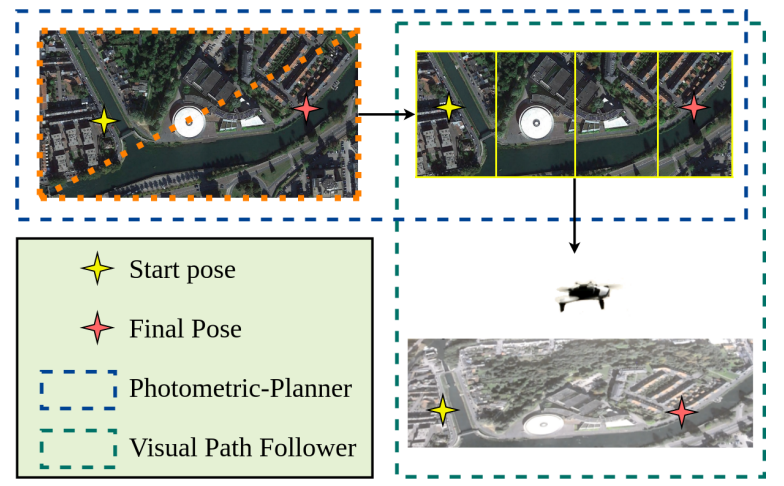

Fig. 1: Proposed vision-based navigation system. Once the start and final poses are defined over the mesh (top left), the Photometric-Planner generates the visual path (top right) to be used for the Visual Path Follower.

\section{Paper Structure}

The rest of the paper follows the next structure. Section III compares current works in the state-of-the-art of visionbased navigation with ours and Section III formally presents the novel Enhanced PVS and highlights two advantages over PVS. Then, Section IV details the process of the PhotometricPlanner and the criterion used to select the key images $K$ among other virtual images $I_{V}$. Later, the Visual Path Follower is detailed in Section $\nabla \mathbf{V}$ as well as the criterion to switch from the current key image $K$ to the next one in the visual path $\mathcal{K}$. The evaluation of the complete system, implemented on the experimental setup, is presented in Section VI The experimentation is composed by a group of positioning and navigation tasks performed over indoor and outdoor environments using an UAV as testbed. Finally, the paper concludes in Section VII by summarizing the main ideas, the interpretation of the results of the experiments and by offering new perspectives for future works.

\section{ReLATEd Works}

This Section reviews the works related to the one presented in this paper. The processes of visual perception and motion control used in [7] are similar of those used in the proposed system since a direct visual feature is considered for the pathplanning and navigation. In this paper we introduce a novel feature, called Gaussian Zero-mean Normalized (GZN) luminance $\check{\mathbf{I}}$, which is a secondary contribution. GZN luminance $\check{\mathbf{I}}$ is a variation of the luminance feature $\mathbf{I}$, being the stacking of every pixel intensities of an image. It is better adapted to compare virtual with onboard images $I_{V}$ and $I$ respectively, and to compute the motion control of the UAV.

In order to accomplish the goal of the Photometric-Planner, the main difficulty is to select the key images $K$ to build the visual path $\mathcal{K}$. As stated in Section $[\mathrm{I}-\mathrm{A}$, a first example to handle this problem is by maximizing common feature points between successive key frames of the visual path [10]. A second one is by means of the visual odometry and SLAMbased approaches, which minimize (relative) pose uncertainty. These approaches had been implemented for the case of ground robot navigation [12], [13] or in the case of a flying robot [14] along the followed path. The last two works are referred as Planning in Information Space, in the more general frame of active perception. In practice, this approach has the implicit behavior of maximizing the number of feature points acquired along the path, a critical constraint for the success of visual odometry and SLAM. However, the PhotometricPlanner addresses the key image selection problem based on the cost $C$ of the Enhanced PVS which directly relates pathplanning with motion control since the same visual feature is used.

The main difficulty of the Visual Path Follower is to compare the key images $K$ with onboard images $I$ using only photometry. This problem had been also solved in the literature [7] where a wheeled robot navigates by comparing the onboard images $I$ from an omnidirectional camera with those stored in the visual path $K$. However, its visual path is obtained from a tele-operated navigation and not from a textured mesh 
$\mathcal{M}$. Another similar approach is the active perception that extends the pose uncertainty minimization-based planning to a photometric criterion [15]. In that approach the robot pose uncertainty is computed as a function of the photometric information of the scene, called Perception-aware Path Planning. However, the system proposed in this paper does not require to build nor update a model while navigating, it rather represents the environment to navigate by a minimum number of key images $K$.

\section{Enhanced Photometric Visual Servoing}

The luminance feature I, used in PVS, is a dense feature more accurate than a sparse one since it stacks pixel intensities of the entire image $I$ to perform a positioning task. However, it is not robust to light variation [5]. Therefore, its implementation to vision-based navigation is restricted to indoor environments only.

Furthermore, the illumination in the textured mesh $\mathcal{M}$ and the scene are unlikely to be the same; thus, PVS is not suitable to compare virtual images $I_{V}$ with onboard images $I$ by the Visual Path Follower. Therefore, a Zero-mean Normalization transformation is applied to the image $I$ in order to make the system robust to global affine illumination changes. Additionally, a Gaussian filtering is also considered to prevent undesired behavior when applying the control input $\mathbf{u}$ to the UAV. The proposed system uses this slight variation of PVS, called Enhanced PVS.

\section{A. Control Law}

The Enhanced PVS considers the GZN luminance feature I which is obtained from the pixel intensities of the image $I$ after a Gaussian filtering with the following kernel:

$$
h(x, y)=\frac{1}{2 \pi \sigma^{2}} e^{-\frac{x^{2}+y^{2}}{2 \sigma^{2}}},
$$

where $\sigma$ is standard deviation, and a Zero-mean Normalization, becoming:

$$
\check{\mathbf{I}}(\boldsymbol{\xi})=\left(\check{\mathbf{I}}_{1 \bullet}, \check{\mathbf{I}}_{2 \bullet}, \ldots, \check{\mathbf{I}}_{N \bullet}\right)^{\top}
$$

where $\check{\mathbf{I}}_{i \bullet} \in \mathbb{R}^{1 \times M}$ is the $i$-th line of the GZN image $\check{I}, N$ is the width and $M$ the height of $\check{I}$.

Then, the GZN photometric error is defined as:

$$
\check{\mathbf{e}}(\boldsymbol{\xi})=\check{\mathbf{I}}(\boldsymbol{\xi})-\check{\mathbf{I}}\left(\boldsymbol{\xi}^{*}\right),
$$

where $\check{\mathbf{I}}(\boldsymbol{\xi})$ and $\check{\mathbf{I}}\left(\xi^{*}\right)$ are obtained from the current and desired GZN images $\check{I}$ and $\check{I}^{*}$, respectively, at the current pose $\boldsymbol{\xi}=\left[\mathbf{X}^{\top}, \boldsymbol{\Theta}^{\top}\right]^{\top} \in \mathbb{R}^{6}$, where $\mathbf{X}=[X, Y, Z]^{\top} \in \mathbb{R}^{3}$ is its location and $\Theta=[\Theta, \Phi, \Psi]^{\top} \in \mathbb{R}^{3}$ its orientation, and desired pose $\boldsymbol{\xi}^{*}$. Analogously to PVS, the goal of Enhanced PVS is to minimize the value of the cost function:

$$
C(\boldsymbol{\xi})=\frac{1}{2}\|\check{\mathbf{e}}(\boldsymbol{\xi})\|^{2},
$$

by non-linear optimization. The chosen control law that handles the motion of the robot is based on the Gauss-Newton method:

$$
\mathbf{u}=-\lambda \widehat{\mathbf{L}}_{\check{\mathbf{I}}\left(\boldsymbol{\xi}^{*}\right)}^{+}\left(\check{\mathbf{I}}(\boldsymbol{\xi})-\check{\mathbf{I}}\left(\boldsymbol{\xi}^{*}\right)\right)
$$

where $\mathbf{u}=\dot{\boldsymbol{\xi}}=\left[\mathbf{v}^{\top}, \boldsymbol{\omega}^{\top}\right]^{\top}$ is the control input composed by linear $\mathbf{v}=\left[v_{x}, v_{y}, v_{z}\right]^{\top}$ and angular $\boldsymbol{\omega}=\left[\omega_{x}, \omega_{y}, \omega_{z}\right]^{\top}$ velocities, $\widehat{\mathbf{L}}_{\mathbf{I}\left(\xi^{*}\right)}^{+}$is the Moore-Penrose pseudoinverse of the approximation of the interaction matrix $\mathbf{L}_{\check{\mathbf{I}}\left(\xi^{*}\right)}=-\nabla \check{\mathbf{I}}\left(\xi^{*}\right)^{\top} \mathbf{L}_{\mathbf{x}}$ related to $\check{\mathbf{I}}\left(\boldsymbol{\xi}^{*}\right), \mathbf{L}_{\breve{\mathbf{I}}\left(\xi^{*}\right)}$ is of full rank, $\nabla \check{\mathbf{I}}\left(\boldsymbol{\xi}^{*}\right)$ is the gradient of $\check{\mathbf{I}}\left(\boldsymbol{\xi}^{*}\right)$ and:

$$
\mathbf{L}_{\mathbf{x}}=\left[\begin{array}{cccccc}
-1 / Z & 0 & x / Z & x y & -\left(1+x^{2}\right) & y \\
0 & -1 / Z & y / Z & 1+y^{2} & -x y & -x
\end{array}\right],
$$

is the interaction matrix that relates the variations of the image point $\mathbf{x}=[x, y]^{\top}$ to the velocity of the camera, as depicted in [16].

Although the Gauss-Newton optimization method is chosen to minimize the cost $C$, the Visual Path Follower is not restricted to any particular method.

\section{B. Cost Function Analysis}

An experimental study shows the main differences between PVS and Enhanced PVS. The study consists into computing the cost functions $C(\boldsymbol{\xi})$ of PVS and Enhanced PVS by rendering virtual images $I_{V}(\boldsymbol{\xi})$ over the image plane around the desired pose $\boldsymbol{\xi}^{*}$. These images are generated by translating $\xi$ along $t_{X}$ and $t_{Y}$ within the intervals of $[-30 \mathrm{~mm}, 30 \mathrm{~mm}]$ in the $X$ and $Y$ axes with a sampling step of $0.5 \mathrm{~mm}$. Fig. 2 2 shows four desired virtual images $I_{V}^{*}$ used in the experimental study of the cost functions $C(\boldsymbol{\xi})$. All of these images are rendered from the textured mesh $\mathcal{M}$ at the desired pose $\boldsymbol{\xi}^{*}$. Notice that the global illumination difference is drastically reduced after applying a Zero-mean Normalization transformation to the obscured $I_{V}^{*}$ with respect to its corresponding non-obscured $I_{V}^{*}$.
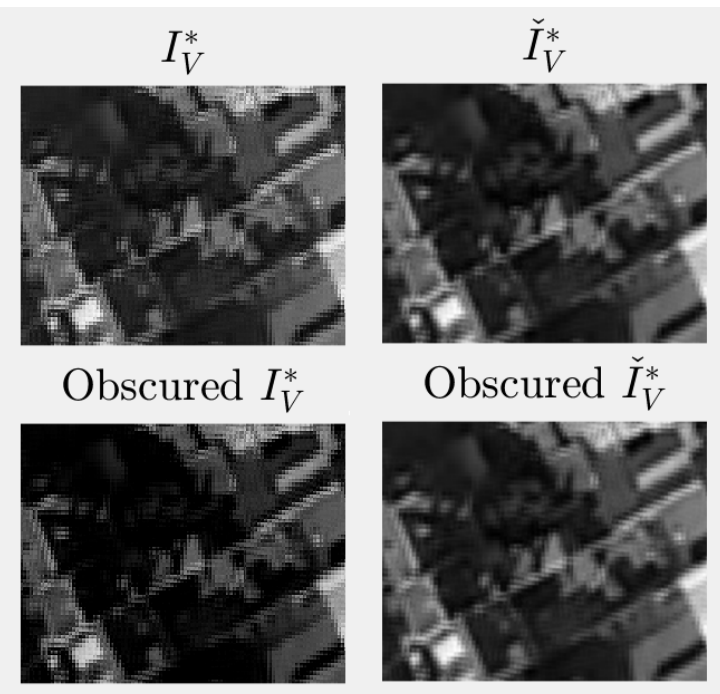

Fig. 2: Four virtual images rendered from the textured mesh at the desired pose for the cost functions study.

Two considerations are made for the experimental study:

- All the feature vectors $\left(\mathbf{I}, \mathbf{I}^{*}, \check{\mathbf{I}}\right.$ and $\left.\check{\mathbf{I}}^{*}\right)$ are generated from virtual images $I_{V}$. The experimental study uses virtual images $I_{V}$ since the Photometric-Planner renders $I_{V}$ from the mesh $\mathcal{M}$. 
- The desired features $\left(\mathbf{I}^{*}\right.$ and $\left.\check{\mathbf{I}}^{*}\right)$ are generated from obscured $I_{V}$ or $\breve{I}_{V}$. This consideration simulates the global illumination difference between the onboard image $I$ and the key image $K$ during the autonomous navigation.

The cost functions $C(\boldsymbol{\xi})$ of PVS, defined as the Sum of Squared Differences (SSD) of pixel intensities, and (4) (GZNSSD) appear in Fig. 3 .

Notice that the value at the minimum of GZNSSD cost function $C(\boldsymbol{\xi})$ reaches zero whereas the corresponding one of SSD does not. Moreover, the shape of the domain of convergence around the global minimum is smoother for GZNSSD which will prevent to compute drastic changes in the input velocity $\mathbf{u}$. Such comparison justifies the choice of Enhanced PVS over PVS for the Photometric-Planner and Visual Path Follower, which are formally introduced in the next two Sections.

\section{PhOtOMETRIC-PlanNer}

The goal of the Photometric-Planner is to generate a navigable visual path $\mathcal{K}$ that allows the Visual Path Follower to reach the final pose $\boldsymbol{\xi}_{f}$ from the start pose $\boldsymbol{\xi}_{s}$. This path contains key images $K=I_{V}\left(\boldsymbol{\xi}_{k}\right) \in \mathcal{K}$ which are rendered from the textured mesh $\mathcal{M}$, using any graphics engine, at key poses $\boldsymbol{\xi}_{k}$. Although several virtual images $I_{V}$ are rendered from the mesh $\mathcal{M}$, just few of them are selected as key images $K$.

The Photometric-Planner begins by defining the start $\boldsymbol{\xi}_{s}$ and final pose $\xi_{f}$ in the textured mesh $\mathcal{M}$. For the sake of simplicity, $\boldsymbol{\xi}_{s}$ and $\boldsymbol{\xi}_{f}$ are considered to have the same orientation $\Theta$. Then, the straight path from $\boldsymbol{\xi}_{f}$ to $\boldsymbol{\xi}_{s}$ is regularly sampled in 3D translation steps $\Delta \mathbf{X}$, into intermediate poses $\boldsymbol{\xi}_{i}$. Indeed, the same orientation $\Theta$ is preserved in $\boldsymbol{\xi}_{i}$ since $\Delta \mathbf{X}$ does not involve rotation. Later, $\boldsymbol{\xi}_{f}$ is considered as the first intermediate pose $\boldsymbol{\xi}_{i}$ and directly selected as the first key pose $\boldsymbol{\xi}_{k}$. Thus, its virtual image $I_{V}\left(\boldsymbol{\xi}_{f}\right)=I_{V}\left(\boldsymbol{\xi}_{k}\right)$ is stored in the visual path $\mathcal{K}$ as the first key image $K$. Analogously, the virtual image rendered from the textured mesh $\mathcal{M}$ at the start pose $I_{V}\left(\boldsymbol{\xi}_{s}\right)$ is considered as the last $K$ in the visual path $\mathcal{K}$. For the rest of the intermediate poses $\boldsymbol{\xi}_{i}$ in the path from

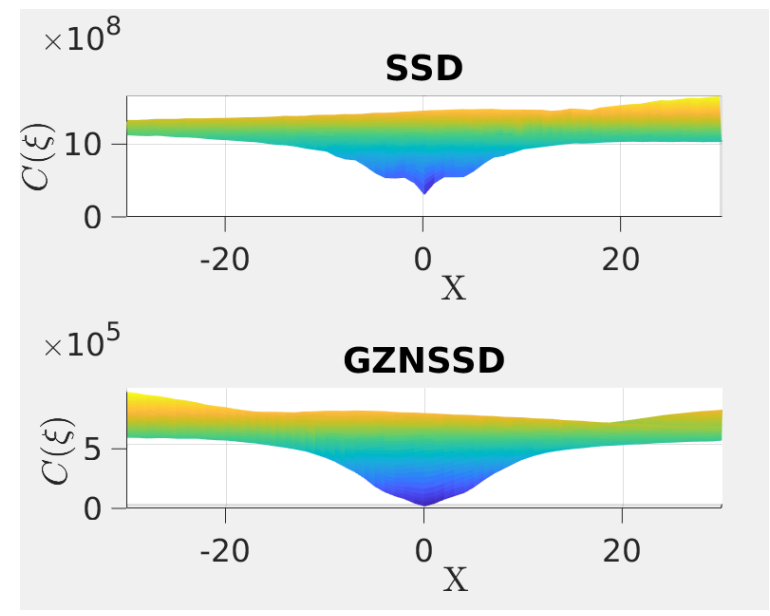

Fig. 3: Comparison of SSD (top) and GZNSSD (bottom) cost functions. Distances are shown in millimeters $\boldsymbol{\xi}_{f}$ to $\boldsymbol{\xi}_{s}$, the selection process is carried out as follows. Let $\boldsymbol{\xi}_{i+1}=\boldsymbol{\xi}_{i}+\Delta \mathbf{X}$ be the next intermediate pose and $\boldsymbol{\xi}_{k}$ the last key pose selected by the Photometric-Planner. Then, $\boldsymbol{\xi}_{i}$ is considered as the next key pose $\boldsymbol{\xi}_{k+1}$, if the cost increase $\Delta C_{i+1}$ falls below a threshold $\epsilon_{s}$ :

$$
\Delta C_{i+1}<\epsilon_{s}
$$

where $\Delta C_{i+1}=C_{i+1}-C_{i}, \quad C_{i}=\frac{1}{2}\left\|\check{\mathbf{I}}\left(\boldsymbol{\xi}_{i}\right)-\check{\mathbf{I}}\left(\boldsymbol{\xi}_{k}\right)\right\|^{2}$, $C_{i+1}=\frac{1}{2}\left\|\check{\mathbf{I}}\left(\boldsymbol{\xi}_{i+1}\right)-\check{\mathbf{I}}\left(\boldsymbol{\xi}_{k}\right)\right\|^{2}$ and the GZN luminance features, $\check{\mathbf{I}}\left(\boldsymbol{\xi}_{i}\right)$ and $\check{\mathbf{I}}\left(\boldsymbol{\xi}_{i+1}\right)$, are obtained from $\check{I}_{V}\left(\boldsymbol{\xi}_{i}\right)$ and $\check{I}_{V}\left(\boldsymbol{\xi}_{i+1}\right)$, respectively (Section III). Generally speaking, such selection criterion does not depend on the image content but it is rather related to the control law convergence.

\section{VISUAL PATH FOLLOWING}

The process of visual path following (Section $[-A$ ) is carried out by the Visual Path Follower, which computes the control input $\mathbf{u}=\dot{\boldsymbol{\xi}}$ from:

$$
\mathbf{u}=-\lambda \widehat{\mathbf{L}}_{\check{\mathbf{I}}\left(\boldsymbol{\xi}_{k}\right)}^{+}\left(\check{\mathbf{I}}(\boldsymbol{\xi})-\check{\mathbf{I}}\left(\boldsymbol{\xi}_{k}\right)\right),
$$

where $\check{\mathbf{I}}\left(\boldsymbol{\xi}_{k}\right)$ is obtained from the key image $K=I_{V}\left(\boldsymbol{\xi}_{k}\right)$ in the visual path $\mathcal{K}$ by transforming $I_{V}$ into $\check{I}_{V}$.

When the current key image $K$ is considered reached during the visual path following, the next key image is set as the current one. This process is called key image update and it occurs when the value of the cost $C$ (4) satisfies: $\left\|\check{\mathbf{I}}(\boldsymbol{\xi})-\check{\mathbf{I}}\left(\boldsymbol{\xi}_{k}\right)\right\|^{2}<\epsilon_{u}$, where $\epsilon_{u}$ is a threshold. The complete process of visual path following is displayed in Fig. 4

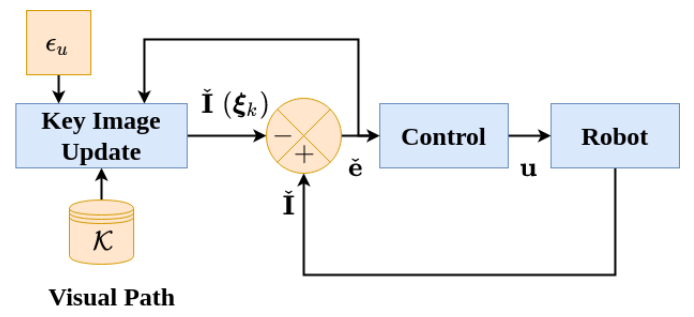

Fig. 4: Visual Path Follower

\section{EXPERIMENTATION}

The performances of the Photometric-Planner and Visual Path Follower are evaluated by navigation tasks. Moreover, since comparing virtual images $I_{V}$ with onboard images $I$ in a visual servoing scheme using an UAV is a novelty, the performance of the Enhanced PVS is evaluated by positioning tasks. Both types of tasks are carried out indoor and outdoor using the experimental setup which is composed by: an HP ZBook 15 computer as ground station, a Parrot Bebop 2 as testbed UAV, the Optitrack motion capture system (indoor) to track the pose $\boldsymbol{\xi}$ of the UAV, a printed tarpaulin (indoor), a Nikon camera to record external video and three textured meshes $\mathcal{M}$. In all the indoor tasks, the same satellite image of $2952 \times 3052$ pixels is printed on the $4.8 \mathrm{~m} \times 4.9 \mathrm{~m}$ tarpaulin and used to generate the textured mesh $\mathcal{M}$. This image is provided by Google Earth [17] and displays a neighborhood of $1146 \mathrm{~m} \times 1170 \mathrm{~m}$ Amiens, France. 
The Bebop 2 is connected to the computer, that runs Ubuntu 16.04, through Wi-Fi using the bebop_autonomy packag ${ }^{1}$ developed for the Robot Operating System [18]. The Photometric-Planner runs offline on the computer using MATLAB and the corresponding textured mesh $\mathcal{M}$ for each navigation task. Indeed, the positioning tasks are an instantiation of the navigation tasks where the visual path $\mathcal{K}$ contains only one key image $K$. The size of all the images is $240 \times 428$ pixels. The Visual Path Follower runs in the computer and sends the control input $\mathbf{u}$ to the UAV. Generally speaking, the onboard images $I$ are acquired by the camera of the Bebop 2 and sent to the ZBook that computes the control input $\mathbf{u}=\dot{\boldsymbol{\xi}}$ to be applied to the robot. However, the Bebop 2 does not obey velocities in the $X$ and $Y$-axes (so, $v_{x}$ and $v_{y}$ ) but angular positions around the $Y$ and $X$-axes (so, $\Phi$ and $\Theta$ ). Fig. 5 . displays the robot and world reference frames ${ }^{R} \mathfrak{F}$ and $W \mathfrak{F}$, respectively.

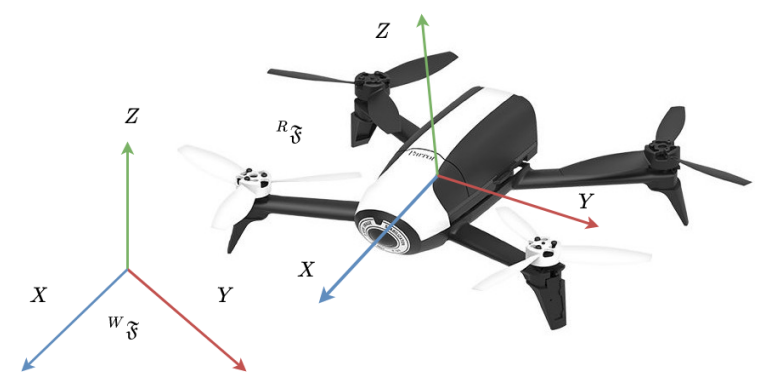

Fig. 5: Reference frames of the robot and the world.

Therefore, a proportional controller is proposed to adapt the output of the control law (8) to the input expected by the Bebop 2. Such controller was computed from a velocity characterization of second order and using the dynamic model of the quadrotor [19] that considers air resistance:

$$
\left[\begin{array}{l}
W^{W} v_{x}(t) \\
{ }^{W} v_{y}(t)
\end{array}\right]=\frac{g}{m}\left[\begin{array}{cc}
c_{0} & 0 \\
0 & c_{1}
\end{array}\right]\left[\begin{array}{l}
\tan \left({ }^{W} \Phi\right)\left(1-e^{-t \frac{c_{0}}{m}}\right) \\
\tan \left({ }^{W} \Theta\right)\left(1-e^{-t \frac{c_{1}}{m}}\right)
\end{array}\right],
$$

where ${ }^{W} v_{x}$ and ${ }^{W} v_{y}$ are the linear velocities in the $X Y$ plane, ${ }^{W} \Phi$ is the pitch and ${ }^{W} \Theta$ is the roll in the world reference frame ${ }^{W} \mathfrak{F}$, the acceleration caused by the gravity is $g=9.8 \mathrm{~m} \cdot \mathrm{s}^{-2}$, the coefficients of the air in both axes are $c_{0}=c_{1}=0.35, t \in \mathbb{R}$ is the time's variable, and the mass of the quadrotor $m=0.497 \mathrm{~kg}$. Nevertheless, such controller is only valid at low speeds. A ProportionalIntegral-Derivative controller [20] would be preferred over a Proportional controller. However, it was not possible to design since the readings of the Inertial Measurement Unit (IMU) are retrieved at a lower rate $(5 \mathrm{~Hz})$ than the control input $(30 \mathrm{~Hz})$ at servoing mode. This issue is related with the update of the Robot Operating System topic that contains the velocity of the Bebop 2 rather than a poor performance of the IMU. This is the main reason to consider the Gaussian filtering in the visual feature.

Furthermore, an image latency, i.e. the time elapsed between an event in the real world and its corresponding change in

\footnotetext{
https: //bebop-autonomy.readthedocs.io/
}

the image on the laptop side, was measured with a mean of $183.9 \mathrm{~ms}$ and a standard deviation of $11.26 \mathrm{~ms}$. The latency was measured using a mbed ${ }^{2}$ microcontroller that triggers a LED and starts a chronometer at the same time. Then, the chronometer stops when a dark pixel, that corresponds to the center of the LED, increments its intensity up to 200 (with a maximum level of 255). In order to mitigate the impact of the latency in the experiments, the robot switches from servoing mode to hovering mode, i.e. to apply a null control input $\mathbf{u}=\mathbf{0}$, during 20 iterations at $30 \mathrm{~Hz}$ when a key image $K$ is reached according to the key image update (Section $\mathrm{V}$ ). Lastly, the tasks are considered failed when the value of the cost $C$ is larger than a threshold $\epsilon_{f}$. The thresholds for the navigation tasks are $\epsilon_{u}=1.5 \times 10^{5}$ for the indoor experiment, $\epsilon_{u}=5.5 \times 10^{4}$ for the outdoor experiment and $\epsilon_{f}=2.75 \times 10^{5}$ for both. All the thresholds are experimentally determined.

The rest of the Section covers the positioning and navigation tasks.

\section{A. Positioning Tasks}

Three positioning tasks evaluate the performance of the Enhanced PVS (Section IIII). The goal of the robot is to servo a desired virtual image $I_{V}^{*}$, using (8) and real images $I$ acquired with its onboard camera.

1) Indoor Nominal Positioning: This experiment consists in servoing a desired virtual image $I_{V}^{*}$ (Fig. 6) without any external perturbation and at constant illumination. It is important to notice that the native hovering mode drifts and the robot eventually diverges since it is based on visual odometry, which is an open-loop system [21].

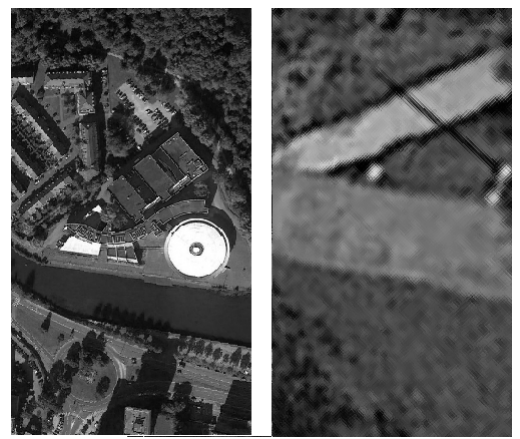

Fig. 6: Desired virtual images used for indoor nominal and perturbed positioning (left) and outdoor positioning (right).

While applying the control law (8), the robot minimizes the cost $C$ (4). Then, the experiment finished when the memory that stores the data of the experiment was filled up in about 5 minutes. The evolution of the cost $C$ and control input $\mathbf{u}$ are displayed in Fig. 7.

2) Indoor Perturbed Positioning: This experiment is similar to the previous one except that a fan is actuated in oscillation mode $2 \mathrm{~m}$ behind the robot to cause external perturbation.

If the native hovering mode is permanently active, the robot diverges in $90 \mathrm{~s}$. However, the Enhanced PVS task lasted $163 \mathrm{~s}$ when a gust of wind pushed the robot strong enough to diverge.

\footnotetext{
${ }^{2}$ Mbed website https://os.mbed.com/
} 


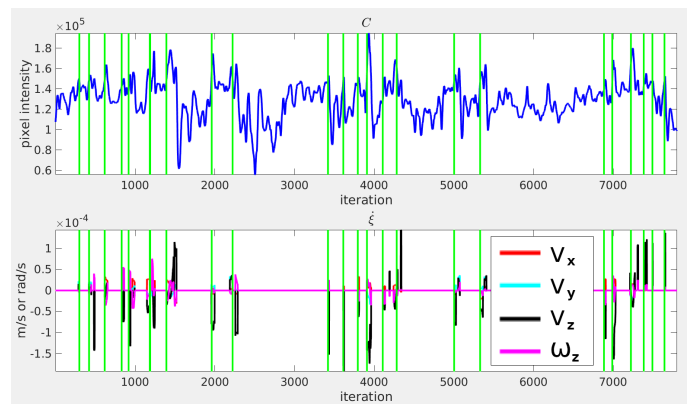

Fig. 7: Cost and control input evolution from the indoor nominal positioning. Vertical lines indicate the switch from hovering mode to servoing mode.

3) Outdoor Positioning: This experiment took place on a crossroad (Fig. 8a) during the day but with presence of clouds. The desired virtual image $I_{V}^{*}$ (Fig. 6) is also generated from the same textured mesh $\mathcal{M}$ as the previous two experiments. The robot stayed in place, at $10 \mathrm{~m}$ above the crossroad, without any important perturbation for the entire task with a duration of $240 \mathrm{~s}$. Indeed, the robot hovered for the most part of the experiment except at the beginning, when servoing for the first time, and at $t=100 \mathrm{~s}$, when a pedestrian caused an increase in the value of the cost $C$ and triggered the servoing mode.

This group of experiments demonstrated that the servoing is performed at scale (concerning image variation and camera displacement) even if $Z$ is not determined from this scheme. Therefore, perturbations that occur at lower altitude had a greater impact in these tasks since they triggered the servoing mode several times whereas perturbations in the image at higher altitude had less impact. The perturbation caused by the pedestrian showed that the Enhanced PVS is robust to partial occlusions. This experiment was conducted with no Global Navigation Satellite System information for guidance and navigation from ground station. Therefore, the quantitative evaluation of this experiment validates the interest of not relying only on Global Navigation Satellite System for robot positioning and navigation.

\section{B. Navigation Tasks}

The performance of the Photometric-Planner and Visual Path Follower is evaluated by two navigation tasks. Although

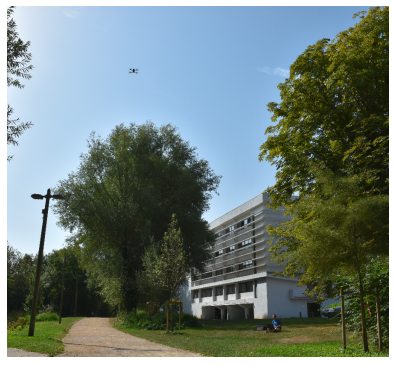

(a) Positioning.

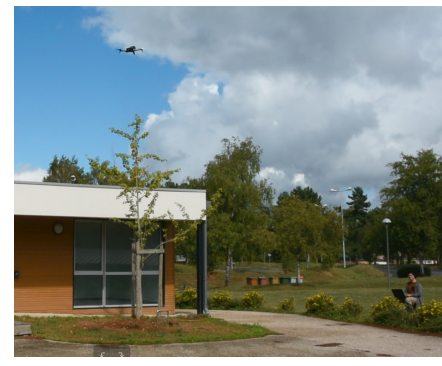

(b) Navigation.
Fig. 8: Outdoor experiments. any set of straight paths could be considered, e.g. an L-path, the motion capture system field of measure constrains the indoor experiment on a single straight path. A straight path is considered outdoor too for consistency. Therefore, to make the path following harder, these tasks are defined as a round trip on a straight line in order to validate the visual path $\mathcal{K}$ in forward and backward directions even if the latter is generated only in backward direction. A video ${ }^{3}$ is provided to present the navigation tasks.

1) Indoor Navigation: In this experiment (Fig. 9), the UAV is intended to travel a straight path of $55 \mathrm{~cm}$. The PhotometricPlanner generated a visual path $\mathcal{K}$ that contains 11 key images $K$ (Fig. 10).

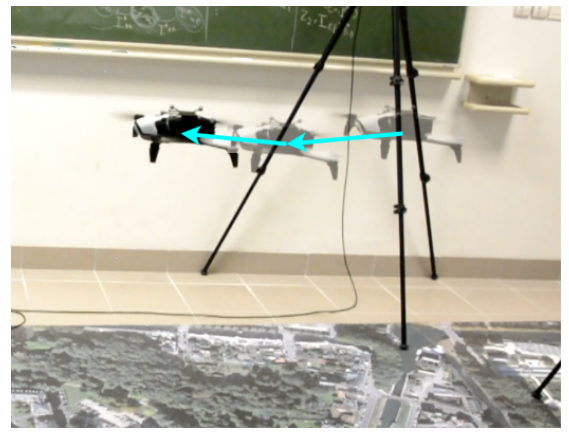

Fig. 9: Indoor navigation experiment. The arrows indicate the trajectory done by the robot at the beginning of the second round trip.

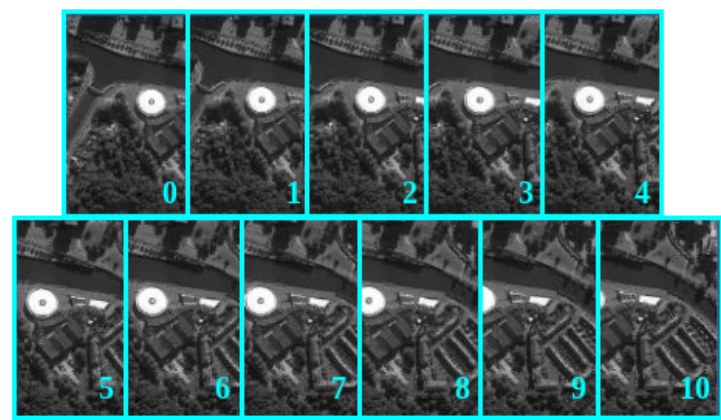

Fig. 10: Visual path of the indoor navigation experiment.

Before diverging, the robot traveled approx. $2.7 \mathrm{~m}$ reaching $54 K:\left\{K_{0}, K_{1}, \ldots, K_{10}\right\},\left\{K_{10}, K_{9}, \ldots, K_{0}\right\}$, $\left\{K_{0}, K_{1}, \ldots, K_{10}\right\}, \quad\left\{K_{10}, K_{9}, \ldots, K_{0}\right\}, \quad$ and $\left\{K_{0}, K_{1}, \ldots, K_{9}\right\}$. Fig. 11 shows the trajectory of the last round trip and Fig. 12 displays the cost $C$ and the control input $\mathbf{u}=\dot{\boldsymbol{\xi}}$ evolution of this experiment. Fig. 13 displays an excerpt of the images of difference between the onboard image $I$ and the key image $K$.

2) Outdoor Navigation: This experiment took place in the car park of the University of Picardie Jules Verne Campus, Amiens, France (Fig. 8b). The textured mesh $\mathcal{M}$ (Fig. 14] is obtained from a snapshot that the UAV took when flying at

3 http://mis.u-picardie.fr/ g-caron/videos/2020 IEEESensors.mp 4 

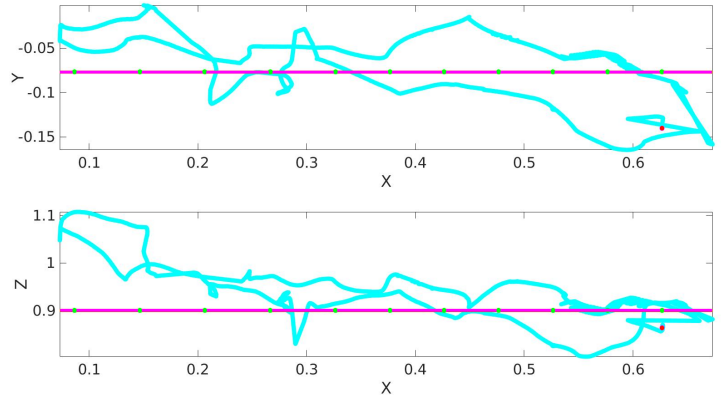

Fig. 11: Last round trip (cyan) of the indoor navigation. Green dots indicate the locations of the key poses over the straight path (pink) and red dots where the system failed. Distances appear in meters.

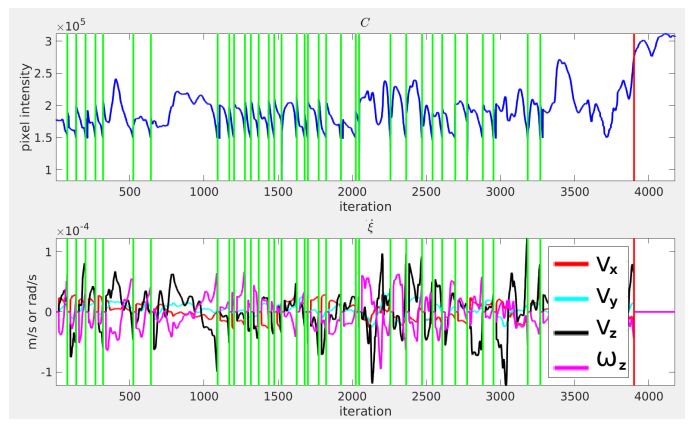

Fig. 12: Cost and control input evolution from the indoor navigation. Vertical lines indicate the key image update and red line when the system failed.

$30 \mathrm{~m}$ above the ground. However, the visual path $\mathcal{K}$ is generated so the robot navigates at a height of $13 \mathrm{~m}$. The distance between the start and final poses $\boldsymbol{\xi}_{s}$ and $\boldsymbol{\xi}_{f}$, respectively, is set to $8 \mathrm{~m}$. The Photometric-Planner generated a visual path $\mathcal{K}$ composed by 5 key images $K$. Then, the robot successfully traveled the round trip, i.e. $16 \mathrm{~m}$, using the visual path $\mathcal{K}$ but it diverged when it tried to restart from the first key image $K$. Fig. 16 shows an excerpt of the images related to this experiment and Fig. 15 displays the evolution of the cost $C$ and the control input $\mathbf{u}=\dot{\boldsymbol{\xi}}$ from this task.

From this group of experiments, the proposed system completed the navigation tasks in round trip but they diverged when an important perturbation in the image or in the robot occurred.

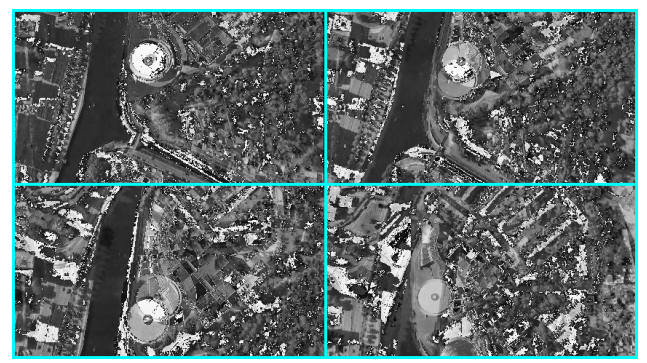

Fig. 13: Excerpt of four images of difference of the indoor navigation experiment.

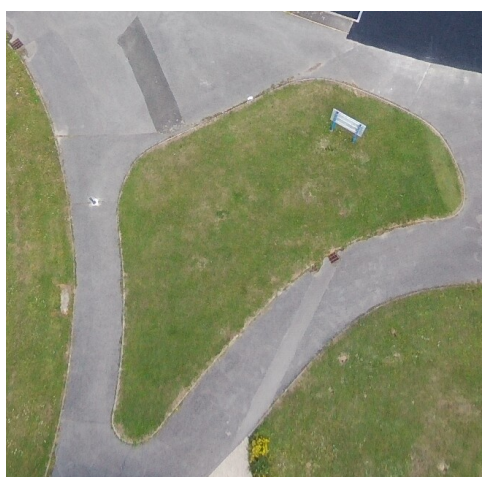

Fig. 14: Textured mesh used for the outdoor navigation.

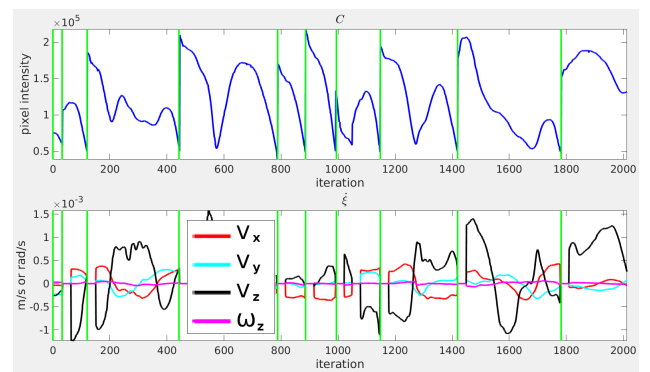

Fig. 15: Cost and control input evolution from the outdoor navigation. Vertical lines indicate the key image update.

\section{CONCLUSIONS}

This paper presented a memory-based navigation system that generates its visual path from a textured mesh rather than from a previous taught path. Therefore, the visual pathplanning process is carried out ex situ and does not require the robot if the textured mesh was obtained a priori. Moreover, such mesh can be instantly generated from a snapshot model [22] as in the last experiment.

The Photometric-Planner generated navigable visual paths validated in both directions and the Visual Path Follower controlled an UAV through a total distance of $16 \mathrm{~m}$ outdoor using only its camera to navigate. In such experiment, navigation based on the Global Navigation Satellite System could not be possible since the signal of the latter was unavailable. Additionally, the Enhanced PVS proved to be able to compare virtual and onboard images while servoing a

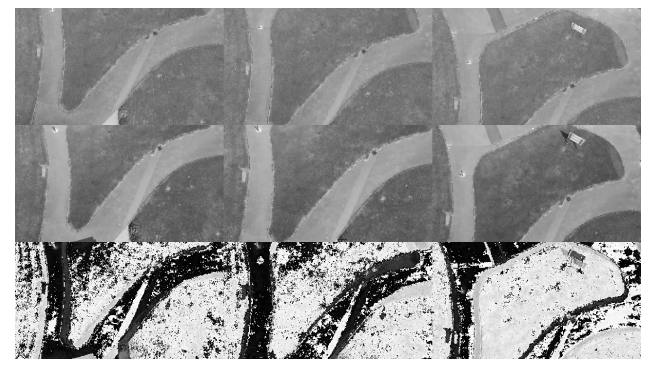

Fig. 16: Excerpt of some images from the outdoor navigation. Desired virtual images appear at the top row, onboard images appear at the central row and images of difference appear at the bottom row. 
commercial UAV. Nevertheless, the Photometric-Planner only validates the navigability of a visual path defined in straight line and does not explore other visual paths. Future works will consider a topological approach to generate paths not strictly straight. Additionally, they will consider another controller, e.g. Model Predictive Control [23], or UAV so the control input be correctly applied and the Visual Path Follower be evaluated in more challenging situations. As a perspective for indoor navigation, the scene model might include obstacles mapped from a group of robots [24] prior to the process of the Photometric-Planner.

\section{ACKNOWLEDGEMENTS}

This research was partially supported by the National Council of Science and Technology (CONACYT) of Mexico.

\section{REFERENCES}

[1] Lu Yuncheng, Xue Zhucun, Xia Gui-Song Xia and Liangpei Zhang, A survey on vision-based UAV navigation, Geo-spatial Information Science, vol. 21, no. 1, pp. 21-32, 2018.

[2] D. Scaramuzza and F. Fraundorfer, Visual Odometry [Tutorial], IEEE Robotics Automation Magazine, vol. 18, no. 4, pp. 80-92, 2011.

[3] G. Bresson and Z. Alsayed and L. Yu and S. Glaser, Simultaneous Localization and Mapping: A Survey of Current Trends in Autonomous Driving, IEEE Trans. on Intelligent Vehicles, vol. 2, no. 3, pp. 194-220, 2017,

[4] P. B. Sujit, S. Saripalli and J. B. Sousa, Unmanned Aerial Vehicle Path Following: A Survey and Analysis of Algorithms for Fixed-Wing Unmanned Aerial Vehicles, IEEE Control Systems, vol. 34, no. 1, pp. 42-59, Feb. 2014.

[5] Raj, Suman and Giordano, Paolo Robuffo and Chaumette, François, Appearance-based indoor navigation by IBVS using mutual information, 2016 Int. Conf. on Control, Automation, Robotics and Vision,

[6] J. Engel and V. Koltun and D. Cremers, Direct Sparse Odometry, IEEE Trans. on Pattern Anal. Mach. Intell, vol. 40. no. 3, pp. 611-625, 2018.

[7] G. Caron, E. Marchand and E. Mouaddib, Photometric visual servoing for omnidirectional cameras, Auton Robot vol. 35, no. 2, 2013, pp. 177-193.

[8] C. Collewet and E. Marchand, Photometric visual servoing, IEEE Trans. on Robotics, vol. 27, no. 4, pp. 828-834, Mar. 2011.

[9] J. Courbon, Y. Mezouar, N. Guénard and P. Martinet, Vision-based navigation of unmanned aerial vehicles, Control Engineering Practice, vol. 18 , no. 7, 2010, pp. 789-799.

[10] A. Remazeilles, and F. Chaumette, Image-based robot navigation from an image memory, Robot. Auton. Syst., Vol. 55, No. 4, 2007, pp. 345356.

[11] Gasteratos, Antonios, et al. "PRONTO: a system for mobile robot navigation via CAD-model guidance." Microprocessors and Microsystems 26.1 (2002): 17-26.

[12] K. Otsu, A. A. Agha-Mohammadi and M. Paton, "Where to Look? Predictive Perception With Applications to Planetary Exploration", IEEE Robot. Autom. Lett., vol. 3, no. 2, 2018, pp. 635-642.

[13] H. Inoue, M. Ono, S. Tamaki and S. Adachi, Active localization for planetary rovers, in IEEE Aerospace Conf. Big Sky, MT, 2016, pp. 1-7.

[14] S. A. Sadat, K. Chutskoff, D. Jungic, J. Wawerla and R. Vaughan, Feature-rich path planning for robust navigation of MAVs with MonoSLAM, in IEEE Int. Conf. on Robotics and Automation, Hong Kong, 2014, pp. 3870-3875.

[15] G. Costante, J. Delmerico, M. Werlberger, P. Valigi, and D. Scaramuzza Exploiting Photometric Information for Planning Under Uncertainty, in Robotics Research. Springer Proceedings in Advanced Robotics vol. 2.

[16] F. Chaumette and S. Hutchinson, Visual servo control. Part I. Basic approaches, in IEEE Robotics Automation Magazine vol. 13, no. 4, pp. $82-90,2006$

[17] Gorelick N., Hancher M., Dixon M., Ilyushchenko S., Thau D. and Moore R., Google Earth Engine: Planetary-scale geospatial analysis for everyone, in Remote sensing of Environment 2017. 1;202:18-27.

[18] Quigley M., Conley K., Gerkey B., Faust J., Foote T., Leibs J., Wheeler R., Ng AY., ROS: an open-source Robot Operating System, in ICRA workshop on open source software 2009. 12 (Vol. 3, No. 3.2, p. 5).
[19] Luukkonen T., Modelling and control of quadcopter, in Independent research project in applied mathematics, Espoo. 2011 22;22.

[20] Salih AL., Moghavvemi M., Mohamed HA., Gaeid KS., Flight PID controller design for a UAV quadrotor, in Scientific research and essays 2010. 4;5(23):3660-7.

[21] Zhang, Ji, and Sanjiv Singh. Visual-lidar odometry and mapping: Lowdrift, robust, and fast, in IEEE Int. Conf. on Robotics and Automation, 2015.

[22] Salichs MA, Moreno L., Navigation of mobile robots: open questions. Robotica. 2000; 18(3): pp. 227-34.

[23] Mercorelli, Paolo, et al. A model predictive control in robotino and its implementation using ros system, in IEEE Int. Conf. on Electrical Systems for Aircraft, Railway, Ship Propulsion and Road Vehicles, 2016.

[24] Sergiyenko, O. Yu, et al. Data transferring model determination in robotic group, in Robotics and Autonomous Systems 2016.

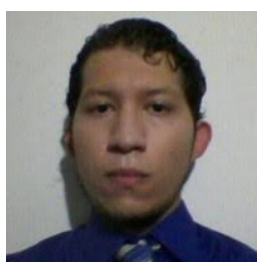

Eder A. Rodríguez Martínez was born in Reynosa Tamaulipas, Mexico. He received the B.S. in Electronic Engineering (2014) and the M.Sc. in electrical Engineering (2016) from the Autonomous University of Tamaulipas (UAT), Mexico, and the PhD in computer science (2020) from the University of Picardie Jules Verne, France.

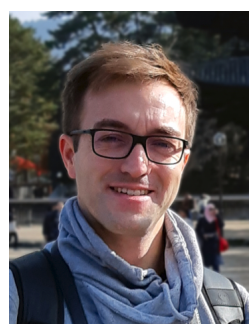

Guillaume Caron Is Associate Professor ("Maître de Conferences") since 2011 and he has been heading the Robotic Perception group of the MIS laboratory since 2016 at Université de Picardie Jules Verne, France. $\mathrm{He}$ received the Ph.D. degree in robotics and the Habilitation degree from the same university in 2010 and 2019. He has been visiting researcher at the CNRS-AIST Joint Robotics Lab, Japan from 2019 to 2021. Before, he stayed at INRIA Rennes, France (2010-2011) and at the University of Osaka, Japan (Apr.-May 2013). His research interests include artificial vision for robotics, real-time visual tracking and servoing.

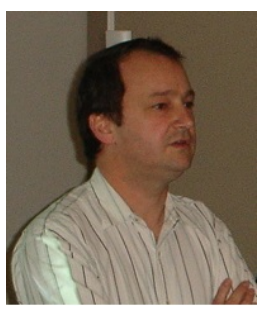

Claude Pégard He received the B.S. in Mechanical and Energy Engineering from the National School of Engineers of Valenciennes, France in June 1984, the DEA in 1984 at the Universite de Valenciennes and the Ph.D. at the Université de Picardie Jules Verne (UPJV) on December 1988. He is research professor at the UPJV since 1989. His research interest area is the control and perception of robotics.

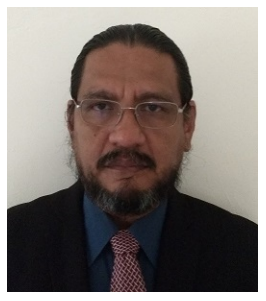

David Lara Alabazares He received the Ph.D. degree in Information and Systems Technologies, with emphasis in control systems, from the University of Technology of Compiègne, France, in 2007. Currently he is a Research Professor at the National Institute of Technology of Mexico, campus Misantla, Veracruz Mexico. His research interests include modeling and control of mechatronic systems, UAVs and renewable energy sources. 